\title{
Entropy Generation Optimization in Internal Combustion Engine
}

\author{
F. Bouras ${ }^{1,2}$ • M. E. H. Attia ${ }^{2}$ - F. Khaldi ${ }^{1}$
}

Received: 28 February 2015 / Accepted: 3 August 2015 /Published online: 12 August 2015

(C) Springer International Publishing Switzerland 2015

\begin{abstract}
In this work, we present a computational investigation of the thermodynamic irreversibilities generated by the combustion process in a cylindrical combustor. The calculations of the turbulent non-premixed combustion properties are based on the aerothermochemical equations. The simulation considers the contribution of each mechanism in the entropy generation sources, i.e., friction, heat conduction, species diffusion and chemical reaction. The computations were carried out by the Fluent-CFD software package. The results show that the chemical reaction and the heat conduction are the major sources of entropy production. Moreover, the fuel preheating reduces significantly thermodynamic irreversibilities in the combustion chamber, while the air factor has a negative effect.
\end{abstract}

Keywords Entropy generation · Turbulent combustion · Diffusion flame - Thermodynamic irreversibility · CFD

\section{Introduction}

It is recognized by many studies that the combustor is the least efficient system in power plants (Markatos and Mukerjee 1981; Shah and Markatos 1987; Stanciu et al. 2007; Khaldi and Adouane 2011). In order to improve the efficiency of the combustion system, it is necessary to identity the physical and chemical mechanisms responsible for thermodynamic irreversibilities. The contribution of each mechanism can be determined by calculating its entropy generation rate, (Liakos et al. 2000; Bejan 2002; Yapici et al. 2005a; Rakopoulos and Giakoumis 2006). Wherefore, the basic equations expressing the distributions of velocity, temperature and species concentrations are used to deduce the local entropy generation rate (Datta 2000; Yapici et al. 2005b; Lior et al. 2006). The review paper of Som and Datta (2008)

F. Bouras

f.bouras@hotmail.fr

1 LPEA, Department of Physics, Faculty of Sciences, University of HL-BATNA, 05000 Batna, Algeria

2 Department of Physics, Faculty of Sciences, University of HL-EL OUED, 39000 Eloued, Algeria 
outlined all the works dealing with computing entropy production in combustion processes covering different fuel types (liquid, gaseous and solid), different configurations (confined, unconfined), different flame types (premixed, non-premixed) and different flow regimes (laminar, turbulent). Laminar flames were considered at first by various researchers (e.g. Datta 2000; Stanciu et al. 2001a; Yapici et al. 2004; Som and Datta 2008) showing that the thermal diffusion, chemical reaction, mass diffusion and the viscous dissipation represent, in order of enumeration, the predominant sources of entropy generation rate. Few works were interested in turbulent flames despite their fundamental role in actual combustors. Stanciu et al. (2001a, 2007) and Yapici et al. (2004, 2005a) works are based on the standard k- $\varepsilon$ model for modeling turbulence and strong irreversible chemical reaction assumptions, i.e., one-step or two-steps exothermic reactions or fast-chemistry reaction. The numerical analysis of Yapici et al. (2004, 2005b) was limited to computing uniquely the thermal and viscous contributions in the entropy generation; the former dominates largely the last. The computation of Stanciu et al. (2001a, 2007) divided the entropy field in mean part and turbulent part. The important result is that at whole the turbulent fluctuations are the major source of entropy generation; in decreasing volumetric rate, the main contributors are chemical, thermal conduction and mass diffusion (Stanciu et al. 2001b, 2007).

The present study is interested in the calculation of thermodynamic irreversibilities occurring in turbulent combustion process in a coaxial jets burner. This combustor configuration was the focus of numerous experimental as well as numerical investigations because of its relatively simple geometry and its representativeness of gas turbine burners. The study is based on the k- $\varepsilon$ turbulence model and it considers a two-step combustion reaction. The local entropy generation rate is calculated taking into account all the mechanisms responsible of entropy generation, i.e., viscous friction, heat conduction, species diffusion and chemical reaction. Besides, the effects of the preheating of the inlet fuel and air factor of mixture on entropy generation are analyzed. All the simulations are carried by the software Fluent.

\section{Burner Configuration}

The present study is a continuation of previous numerical investigations of Bouras et al. (2010, 2012) based on the experimental work of Owen et al. (1976), where the interest was on turbulent diffusion flame characterization in the coaxial jet combustor configuration (Owen et al. 1976; Pierce and Moin 2004). The burner depicted in Fig. 1 and considered in the experimental study consists of a coaxial jet discharging into a cylindrical chamber pressurized

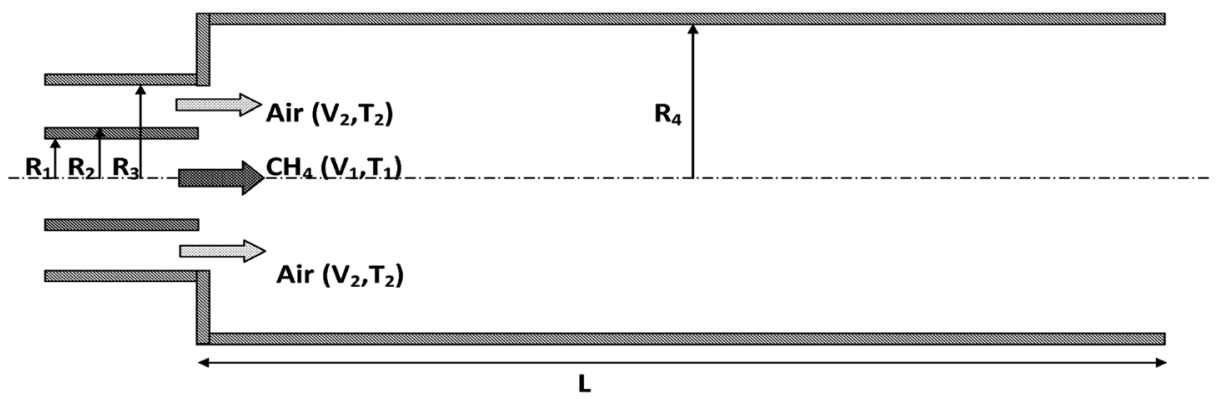

Fig. 1 Schematic of the burner 
to $3.8 \mathrm{~atm}$. The burner is of radius $\mathrm{R}_{4}=0.06115 \mathrm{~m}$ and length $\mathrm{L}=1 \mathrm{~m}$ and is with isothermal walls of $500 \mathrm{~K}$. The fuel $\left(\mathrm{CH}_{4}\right)$ is issued from the inner jet with radius $\mathrm{R}_{1}=0.03157 \mathrm{~m}$, at a velocity $\mathrm{V}_{1}=0.987 \mathrm{~m} / \mathrm{s}$ and at temperature $\mathrm{T}_{1}=300 \mathrm{~K}$, while the preheated air at temperature $\mathrm{T}_{2}=750 \mathrm{~K}$ is supplied from the annular jet of external radius $\mathrm{R}_{3}=0.04685 \mathrm{~m}$ at a velocity $V_{2}=$ $20.63 \mathrm{~m} / \mathrm{s}$.

This work consists a two-dimensional (2D) study of the entropy generation in diffusion flame. We produced the geometry and meshes by GAMBIT, and the chosen meshes are of quadrilateral type, where the selected grid is smoothed in the region nearby the solid walls of the burner and at the level of interaction of air and $\mathrm{CH}_{4}$, in order to accommodate more information in solid boundary and flame zone. The volume contained approximately 40.000 cells, the size of a cell ranged from $4.87 \mathrm{E}-09 \mathrm{~m}^{2}$ to $1.52 \mathrm{E}-04 \mathrm{~m}^{2}$.

\section{Governing Equations}

The simulation of combustion in the burner is assumed steady, turbulent and axisymmetric. According to these assumptions, the set of equations suitable for the adopted combustion model are the following (Stanciu et al. 2001a; Yapici et al. 2005a; Stanciu et al. 2007; Som and Datta 2008):

$$
\text { Continuity : } \quad \frac{\partial}{\partial x_{i}}\left(\rho u_{i}\right)=0
$$

and: $\rho:$ Density; $u_{i}$ : velocity components.

$$
\text { Momentum } \frac{\partial}{\partial x_{i}}\left(\rho u_{i} u_{j}\right)=-\frac{\partial p}{\partial x_{j}}+\frac{\partial \tau_{i j}}{\partial x_{j}}
$$

and: $u_{j}$ : velocity components; $p$ : Pressure; $\tau$. Shear stress tensor; $x_{i}$ : Cartesian coordinate.

$$
\text { Energy : } \quad \frac{\partial}{\partial x_{i}}\left(\rho u_{i} h\right)=u_{i} \frac{\partial p}{\partial x_{i}}+\tau_{i j} \frac{\partial u_{i}}{\partial x_{j}}+\frac{\partial}{\partial x_{i}} \lambda_{\text {eff }} \frac{\partial T}{\partial x_{i}}-\frac{\partial}{\partial x_{i}}\left(\sum_{k=1}^{n} \rho y_{k} U_{k, i} h_{k}\right)
$$

and: $h$ : Enthalpy; $T$ : Temperature; $\lambda$ : Thermal conductivity; $y$ : Mass fraction of chemical species.

$$
\text { Species : } \quad \frac{\partial}{\partial x_{i}}\left(\rho u_{i} y_{k}\right)=\frac{\partial^{2} \rho D_{k} y_{k}}{\partial x^{2}{ }_{i}}+\dot{\omega}_{k}
$$

and: $D$ :Coefficient of mass diffusion; $\omega:$ Arrhenius terms.

$$
\text { Thermodynamic status : } \quad p=\rho R T \sum_{k=1}^{n} \frac{y_{k}}{M_{k}}
$$

and: $M_{k}$ :Mass Molar; $R$ : Constant of ideal gas.

Turbulence kineticenergy, $k: \quad \frac{\partial}{\partial x_{i}}\left(\rho u_{i} k\right)=\frac{\partial}{\partial x_{j}}\left(\mu+\frac{\mu_{t}}{\sigma_{k}}\right) \frac{\partial k}{\partial x_{j}}+G_{k}-\rho \varepsilon+S_{k}$

Dissipationrate, $\varepsilon: \quad \frac{\partial}{\partial x_{i}}\left(\rho u_{i} \varepsilon\right)=\frac{\partial}{\partial x_{j}}\left(\mu+\frac{\mu_{t}}{\sigma_{\varepsilon}}\right) \frac{\partial \varepsilon}{\partial x_{j}}+C_{1 \varepsilon} \frac{\varepsilon}{k} G_{\varepsilon}-C_{2 \varepsilon} \rho \frac{\varepsilon}{k}+S_{\varepsilon}$ 
where: $\mu$ : Viscosity and $C_{1 \varepsilon}=1.44, C_{2 \varepsilon}=1.92, \sigma_{k}=1.0, \sigma_{\varepsilon}=1.3$ are k- turbulence model constant.

The combustion reaction is modeled with a two-step reaction mechanism where the production and the combustion of carbon monoxide $(\mathrm{CO})$ are taken into account. In the first stage, methane is oxidized into carbon monoxide and water vapor, and in the second, carbon monoxide is oxidized into carbon dioxide. The reaction mechanism takes place according to the constraints of chemistry, and is defined by stoichiometric equations (Som and Datta 2008; Yapici et al. 2004):

$$
\begin{aligned}
& \text { Reaction } 1: \quad \mathrm{CH}_{4}+\frac{3}{2} \mathrm{O}_{2} \rightarrow \mathrm{CO}+2 \mathrm{H}_{2} \mathrm{O} \quad \text { (step 1) } \\
& \text { Reaction 2: } \quad \mathrm{CO}+\frac{1}{2} \mathrm{O}_{2} \rightarrow \mathrm{CO}_{2} \quad \text { (step 2). }
\end{aligned}
$$

Once the computations are made, the results obtained are exploited to calculate the local entropy generation rate, as follows (Stanciu et al. 2001b; Rakopoulos and Kyritsis 2001; Yapici et al. 2005b; Som and Datta 2008):

$$
\dot{S}_{g e n}=\left(\dot{S}_{g e n}\right)_{f}+\left(\dot{S}_{g e n}\right)_{h}+\left(\dot{S}_{g e n}\right)_{d}+\left(\dot{S}_{g e n}\right)_{c h}
$$

where:

$$
\left(\dot{S}_{g e n}\right)_{f}: \text { Fluid friction volumetric entropy generation rate, }\left(\dot{S}_{g e n}\right)_{f}=\frac{\mu_{e f f}}{T} \frac{\partial u_{i}}{\partial x_{j}} \frac{\partial u_{i}}{\partial x_{j}}>0
$$

$$
\left(\dot{S}_{g e n}\right)_{h}: \text { Heat transfer volumetric entropy generation rate, }\left(\dot{S}_{g e n}\right)_{h}=\frac{\lambda_{\text {eff }}}{T^{2}} \frac{\partial T}{\partial x_{j}} \frac{\partial T}{\partial x_{j}}>0
$$

$\left(\dot{S}_{g e n}\right)_{d}$ : Diffusion of species volumetric entropy generation rate, $\left(\dot{S}_{g e n}\right)_{d}=\sum_{k=1}^{n} \rho D_{k} \frac{R_{k}}{y_{k}} \frac{\partial y_{k}}{\partial x_{i}} \frac{\partial y_{k}}{\partial x_{i}}>0$

$$
\left(\dot{S}_{g e n}\right)_{c h}: \text { Chemical reaction volumetric entropy generation rate, }\left(\dot{S}_{g e n}\right)_{c h}=\frac{\dot{\omega}}{T} \sum_{k=1}^{n}\left(\gamma_{k}^{\prime}-\gamma_{k}^{\prime \prime}\right) \mu_{k}>0
$$

and: $\mu_{k}$ : Chemical potential; $\gamma$ : Stoichiometric coefficient.

We used FLUENT-CFD package to resolve the governing equations mentioned previously. We note that the SIMPLEC algorithm is used for pressure velocity coupling. And, the convergence criteria for solving equations are equal to $10^{-3}$. This investigation shows two types of results. The first one is the validation of computational profiles of axial velocity and 
temperature field with experimental data for methane fueled coaxial jet combustor (Owen et al. 1976; Pierce and Moin 2004), and the second one, the obtained results are exploited to evaluate the entropy generation rate for different cases of flows.

\section{Results and Discussion}

\subsection{Numerical Validation}

The primary quantities were tested against experimental data that used the length $\mathrm{R}$ and the velocity $U$ normalized by the injector radius $\left(R \equiv R_{3}\right)$ and the inlet bulk velocity of air $\left(U \equiv V_{2}\right)$.

\subsubsection{Axial Velocity}

Figure 2 presents the comparison of computational and experimental radial profile of axial velocity. Indeed, the velocity fields achieve significantly better agreement with the experiment at two measurement stations $x / R=0.14$ and $x / R=4.67$. The mean axial velocity ranges from about $u / U=-0.15$ to 1.1 , where the maximum is located in the flame zone. The negative values of velocity are present close to the wall in the recirculating region and in the center of the burner: a brutal variation in the section of the burner results in the creation of the recirculating zones what can be explained by the significant negative values observed close to the wall. In addition, the difference between the inlet velocity of $\mathrm{CH}_{4}$ and air produces the second zone of recirculation, which appears at the center of the combustion chamber. The meeting of the two inlet flows is characterized by high mixture level generated by the shearing where the flame stabilized in this region (Bouras et al. 2010, 2012; Pierce and Moin 2004). The zone of the flame is the seat of the great values of velocity. In particular, the position of peak velocity is reproduced well by the present numerical computation. Furthermore, in the recirculating region and the flame holder, the agreement is satisfactory.

\subsubsection{Temperature}

The comparisons of the predicted radial profiles of temperature with those of experiments at two measurement stations, $x / R=4.52$ and $x / R=5.20$, are shown in Fig. 3. Temperature is a quantity that is derived from the energy equation by assuming isothermal walls and neglecting thermal radiation. The high values of the temperature are located within the reaction zone around the center of the burner; away the temperature decreases to achieve the wall temperature. Indeed, the numerical temperature profiles give similar experimental trends but with overestimation, especially near the center of the burner. The expected overprediction of temperature can be attributed to three factors: i) ignoring of thermal radiation in the numerical simulation, ii) experimental uncertainty, especially in regions with large temperature fluctuations, and iii) difficulty to ensure perfectly the experiment condition of isothermal water-cooled walls at 500K (Bouras et al. 2010, 2012; Pierce and Moin 2004).

\subsection{Entropy Generation Analysis}

Figure 4 illustrates the pie chart of the entropy generation rates produced by all the mechanisms involved in the combustion process. The total generated entropy is about $140 \mathrm{~W} / \mathrm{K}$. 

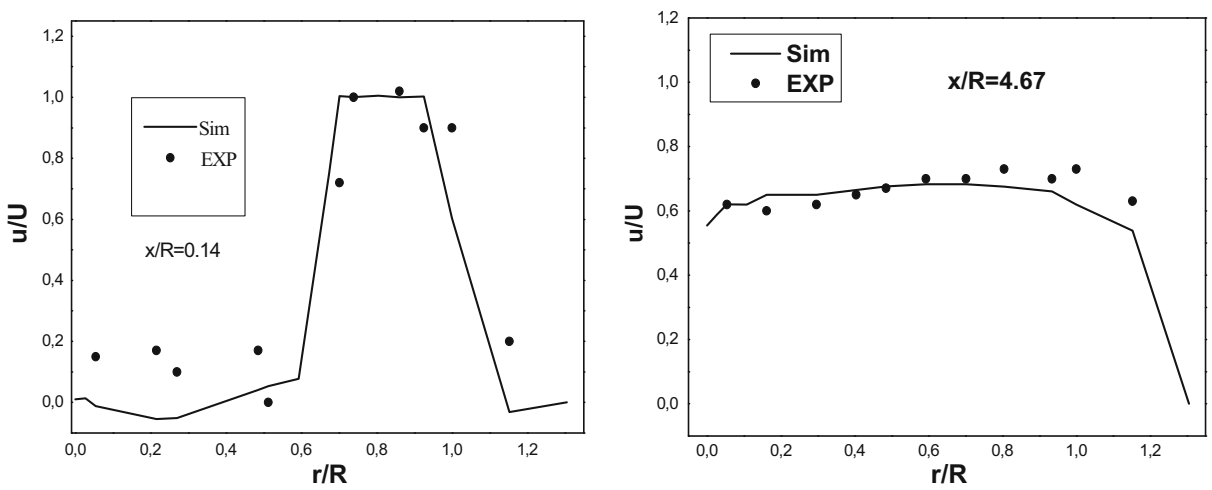

Fig. 2 Radial profiles of normalized axial velocity

Chemical reaction (52\%) is the first producer of entropy. In the second order comes the heat conduction which is responsible of $38 \%$. Therefore, species diffusion transfers have moderate part $(10 \%)$. The effect of viscosity friction is negligible regarding the generation of thermodynamic irreversibilities.

\subsubsection{Effect of Preheating Fuel on Entropy Generation}

In this part of work, we performed a detailed numerical analysis for the study of entropy generation considering the preheated $\mathrm{CH}_{4}$ at the inlet, using the mathematical equations presented previously (Eqs. 9, 10, 11, 12 and 13). Figure 5 illustrates the obtained entropy generation sources rates for different $\mathrm{CH}_{4}$ inlet temperatures. It has been shown that the total rate of entropy generation becomes large in the region of high temperature gradient. The chemical reaction accounted for the maximum entropy generation in $\mathrm{CH}_{4}$ flame, while thermal diffusion contributed in the second largest one after the entropy generation due to the chemical reaction. The contribution of diffusion species gives less impact comparatively to two previous sources. However, the effect of the viscous dissipation on the total entropy generation is negligible. Moreover, the preheated fuel in a stoichiometric, $\mathrm{CH}_{4}$-air, flame has given a reduction in the total entropy generation rate by approximately $0.4 \%$ with the difference of
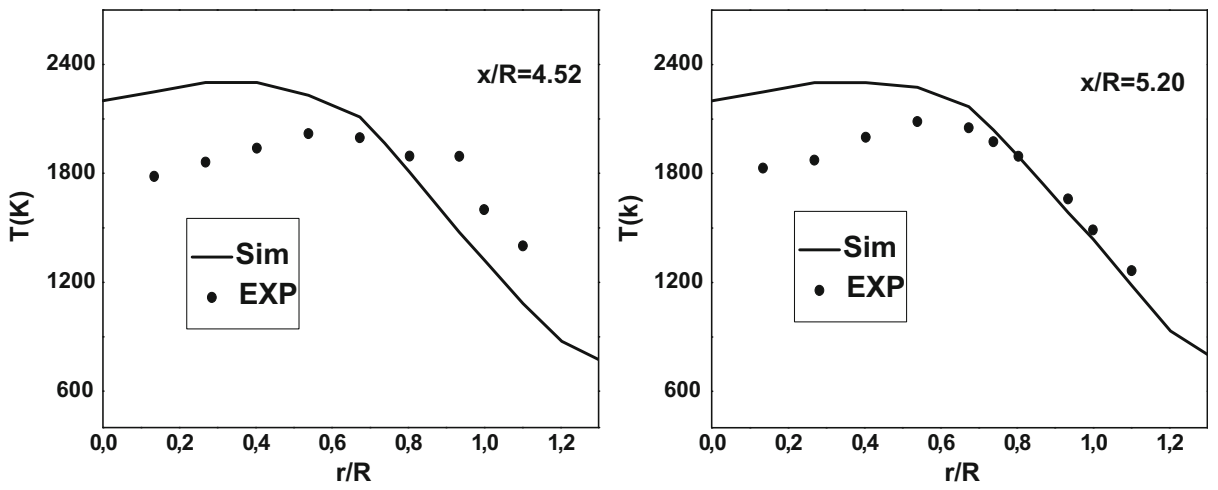

Fig. 3 Radial profiles of Temperature 


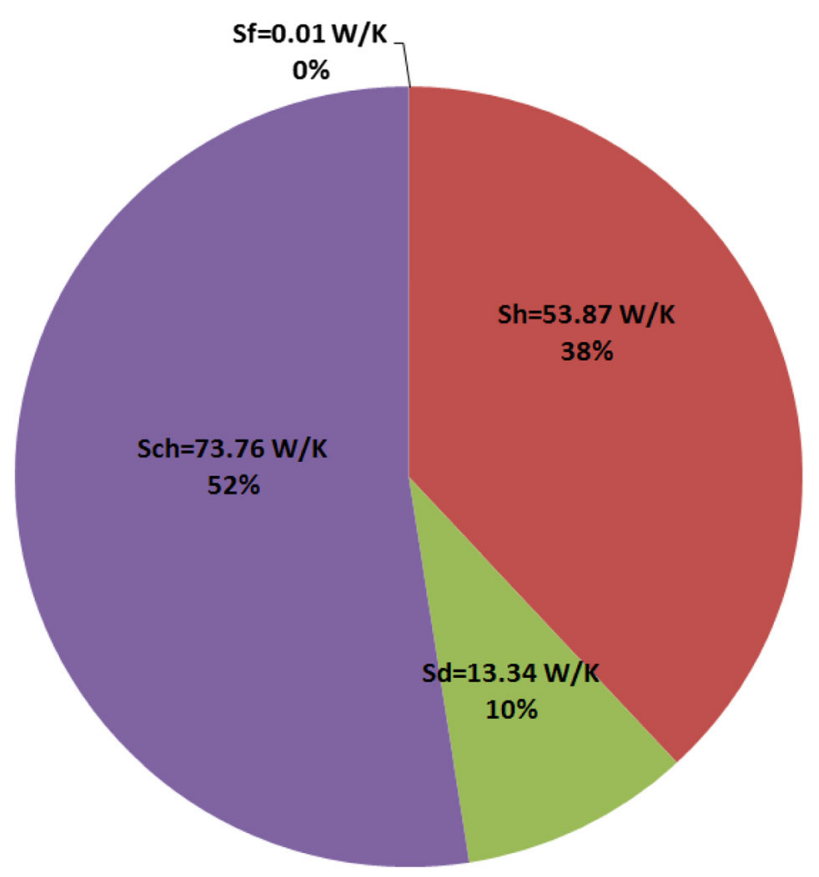

Fig. 4 Pie charts of entropy generation

$10^{\circ} \mathrm{C}$ of temperature relatively to the reference flow. However, the effect of the preheated fuel on the total entropy generation was found to be much smaller, and the effect of the viscous dissipation negligible. So, the total entropy generation decreases with the increase of inlet temperature of fuel $\left(\mathrm{CH}_{4}\right)$.

\subsubsection{Effect of air Factor on Entropy Generation}

Air factor, $\lambda$, is defined as the relation between the actual air fuel ratio and the stoichiometry air fuel ratio. Based on this definition, we can distinguish the type of the reactive mixture: $\lambda=1.0$ is at stoichiometry, rich mixtures $\lambda<1.0$, and lean mixtures $\lambda>1.0$. In this section, the effect of air factor on the creation of thermodynamic irreversibilities in the combustion chamber is analyzed for each process of entropy generation contribution as shown in Fig. 6. The air factor, $\lambda$, varies from 0.5 to 1.3 . The combustors of gas turbines run always with air excess regime where the air factor is greater than $1(\lambda>1)$. The first result is that the total entropy generated by the combustion process increases with increasing air factor, in deficit air as well as in excess air regimes. Entropy production due to heat conduction increases monotonously over all the range of air factor. The variation is more rapid in the air excess regime. The entropy generation caused by chemical reaction has two trends; at first, it increases in the air deficit regime $(\lambda<1)$ and then decreases slowly in the air excess regime. The increasing entropy generation due to heat conduction and that due to the decreasing chemical reaction meet at air factor equal to 1.3. Moreover, in air excess regime the burner is diluted by air, noncombustible. Thus, an amount of heat combustion released in the burner is absorbed by nonparticipating matter in the combustion reaction. This causes the average level of flame temperature falling lower than 


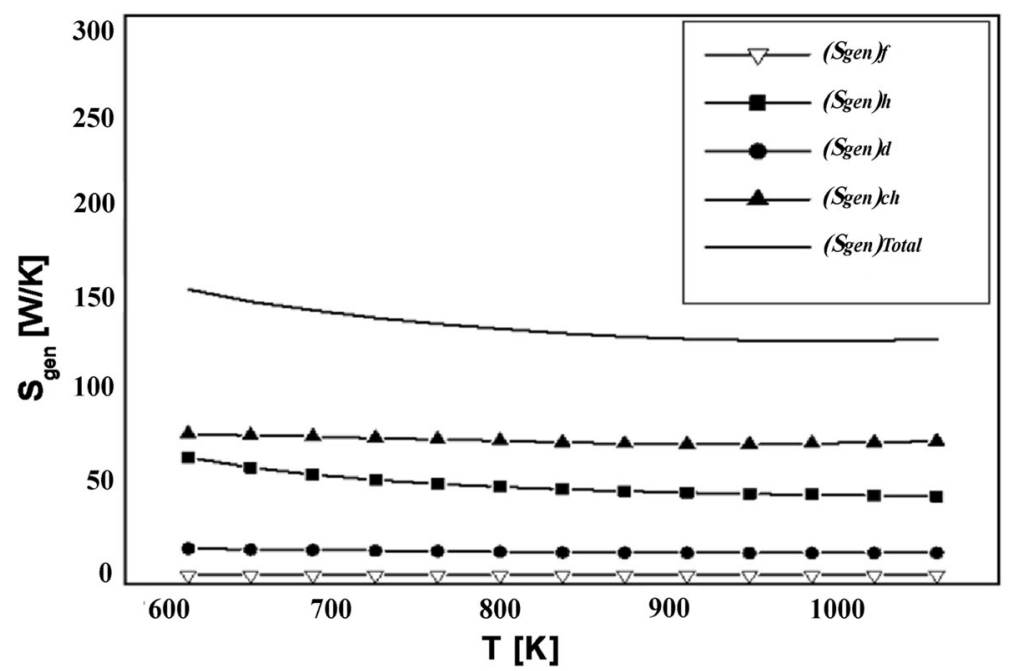

Fig. 5 Effect of preheating fuel on entropy generation

its level obtained at stoichiometry fuel/air mixture. Among all the considered mechanisms, entropy generation due to heat conduction is more sensible versus air factor; it shows a more rapid variation because its dependency to temperature squared, in accordance with Eq. (11).

\section{Conclusions}

The aim of the paper is the assessment of the thermodynamic inefficiencies occurring during combustion processes. Through entropy generation analysis, we were able to identify the role of every physical and chemical mechanism involved in generating thermodynamic

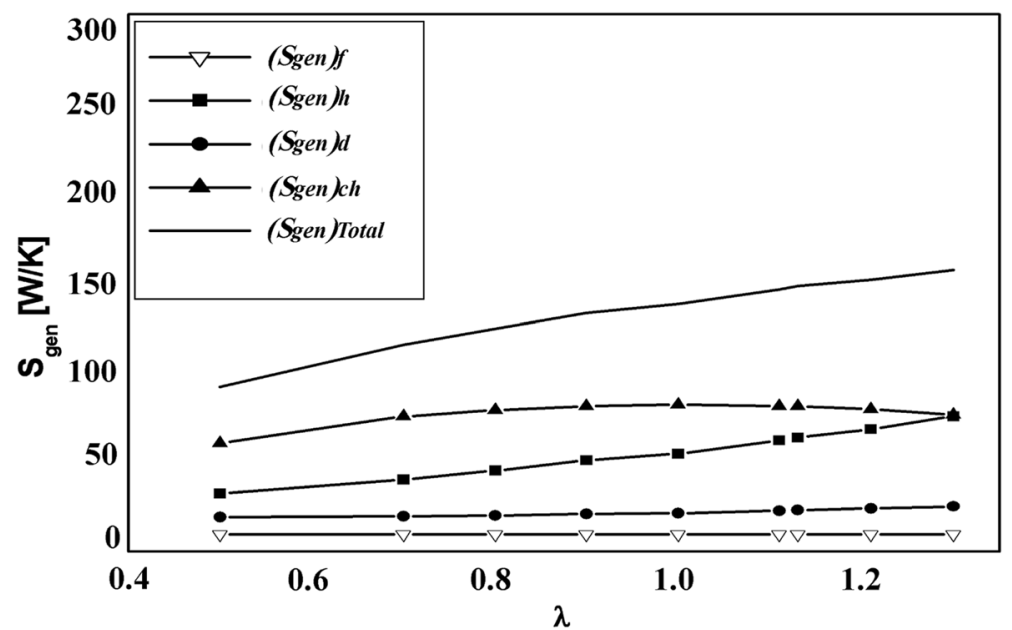

Fig. 6 Effect of air factor on entropy generation 
irreversibilities. The analysis is based on the numerical simulation, by the software Fluent, of a typical combustion system. The case study consists of a turbulent diffusion flame burning in a cylindrical burner; a configuration representative of gas turbine combustors. At first, the results, in terms of velocity, temperature and species fields are compared to experimental reference data. Then, it appears that chemical reaction and heat transfer are the more important processes responsible for thermodynamic irreversibilities; they are responsible, respectively, for 50 and $40 \%$ of entropy generation rate. The species diffusion has a moderate role, around $10 \%$, while the irreversibilities generated by viscous friction are negligible. Preheating of the inlet fuel has a positive impact on the total thermodynamic irreversibilities associated to the combustion process by reducing the entropy generation rate due to heat transfer. Air excess has negative impact on the efficiency of combustion processes. It increases considerably the share and dominance of heat conduction in generating entropy.

Acknowledgments An initial version of this paper has been presented at the International Conference on Integrated Management of Environment (http://www.icime.net/), 25-28 September 2014, Hammamet, Tunisia.

\section{References}

Bejan A (2002) Fundamentals of exergy analysis, entropy generation minimization, and the generation of flow architecture. Int J Energy Res 26:545-565

Bouras F, Soudani A, Si Ameur A (2010) Beta-pdf approach for large-eddy simulation of non-premixed turbulent combustion. Int Rev Mechanical Eng 4:358-363

Bouras F, Soudani A, Si Ameur A (2012) Thermochemical study of Internal Combustion Engine. Energy Procedia 18:1086-1095

Datta A (2000) Entropy generation in a confined laminar diffusion flame. Combust Sci Technol 159:39-56

Khaldi F, Adouane B (2011) Energy and exergy analysis of a gas turbine power plant in Algeria. Int J Exergy 9: 399-413

Liakos HH, Founti MA, Markatos NC (2000) The relative importance of combustion mechanisms in industrial premixed flames under high pressure. Appl Therm Eng 20:925-940

Lior N, Sarmiento-Darkin W, Al-Sharqawi HS (2006) The exergy fields in transport processes: their calculation and use. Energy 31:553-578

Markatos NC, Mukerjee T (1981) Three-dimensional Computer Analysis of Flow and Combustion in Automotive Internal Combustion Engines. Mathematics and Computers in Simulation. Transactions IMACS 23:354-366

Owen FK, Spadaccini LJ, Bowman CT (1976) Pollutant formation and energy release in confined turbulent diffusion flames. Proc Combust Inst 16:105-117

Pierce CD, Moin P (2004) Progress-variable approach for large-eddy simulation of non-premixed turbulent combustion. J Fluid Mech 504:73-97

Rakopoulos CD, Giakoumis EG (2006) Second-law analyses applied to internal combustion engines Operation. Prog Energy Combust Sci 32:2-47

Rakopoulos CD, Kyritsis DC (2001) Comparative second-law analysis of internal combustion engine operation for methane, methanol, and dodecane fuels. Energy 26:705-722

Shah P, Markatos NC (1987) Computer simulation of turbulence in internal combustion engines. Int J Numerical Methods fluids 7:927-952

Som SK, Datta A (2008) Thermodynamic irreversibilities and exergy balance in combustion processes. Prog Energy Combust Sci 34:351-376

Stanciu D, Isvoranu D, Marinescu M (2001a) Entropy generation rates diffusion flames. Int J Applied Thermodynamics 1:1-18

Stanciu D, Isvoranu D, Marinescu M, Gogus Y (2001b) Second law analysis of diffusion flames. Int J Appl Thermodynamics 4:1-18

Stanciu D, Marinescu M, Dobrovicescu A (2007) The influence of swirl angle on the irreversibilities in turbulent diffusion flames. Int J Thermodynamics 10:143-153 
Yapici H, Basturk G, Kayatas N, Albayrak B (2004) Numerical study of effect of oxygen fraction on local entropy generation in a methane-air burner. Sadhana 29:641-667

Yapici H, Kayatas N, Albayrak B, Basturk G (2005a) Numerical calculation of local entropy generation in a methane-air burner. Energy Convers Manage 46:885-919

Yapici H, Kayatas N, Albayrak B, Basturk G (2005b) Numerical study on local entropy generation in a burner fueled with various fuels. Heat Mass Transfer 41:519-534 